\title{
Some combinatorial arrays related to the Lotka-Volterra system
}

\author{
David Callan \\ Department of Statistics \\ University of Wisconsin-Madison \\ Madison, WI 53706, U.S.A. \\ callan@stat.wisc.edu
}

Shi-Mei Ma*

School of Mathematics and Statistics Northeastern University at Qinhuangdao Hebei 066004, P.R. China

shimeimapapers@163.com

\author{
Toufik Mansour \\ Department of Mathematics \\ University of Haifa \\ 3498838 Haifa, Israel \\ tmansour@univ.haifa.ac.il
}

Submitted: May 30, 2014; Accepted: May 6, 2015; Published: May 14, 2015

Mathematics Subject Classifications: 05A05, 05A18

\begin{abstract}
The purpose of this paper is to investigate several context-free grammars suggested by the Lotka-Volterra system. Some combinatorial arrays, involving the Stirling numbers of the second kind and Eulerian numbers, are generated by these context-free grammars. In particular, we present grammatical characterization of some statistics on cyclically ordered partitions.
\end{abstract}

Keywords: Lotka-Volterra system; Context-free grammars; Cyclically ordered partitions; Eulerian numbers

\section{Introduction}

Throughout this paper a context-free grammar is in the sense of Chen [4]: for an alphabet $A$, let $\mathbb{Q}[[A]]$ be the rational commutative ring of formal power series in monomials formed from letters in $A$. A context-free grammar over $\mathrm{A}$ is a function $G: A \rightarrow \mathbb{Q}[[A]]$ that replace

*The second author is responsible for all the communications and he was supported by NSFC (11401083), the Fundamental Research Funds for the Central Universities (N130423010), the Research Foundation for Science and Technology Pillar Program of Northeastern University at Qinhuangdao (XNK201303) and the Natural Science Foundation of Hebei Province (A2013501070). 
a letter in $A$ by a formal function over $A$. The formal derivative $D$ is a linear operator defined with respect to a context-free grammar $G$. More precisely, the derivative $D=D_{G}$ : $\mathbb{Q}[[A]] \rightarrow \mathbb{Q}[[A]]$ is defined as follows: for $x \in A$, we have $D(x)=G(x)$; for a monomial $u$ in $\mathbb{Q}[[A]], D(u)$ is defined so that $D$ is a derivation, and for a general element $q \in \mathbb{Q}[[A]]$, $D(q)$ is defined by linearity.

Let $[n]=\{1,2, \ldots, n\}$. The Stirling number of the second kind $\left\{\begin{array}{l}n \\ k\end{array}\right\}$ is the number of ways to partition $[n]$ into $k$ blocks. Let $\mathfrak{S}_{n}$ be the symmetric group of all permutations of $[n]$. A descent of a permutation $\pi \in \mathfrak{S}_{n}$ is a position $i$ such that $\pi(i)>\pi(i+1)$. Denote by $\operatorname{des}(\pi)$ the number of descents of $\pi$. The Eulerian number $\left\langle\begin{array}{l}n \\ k\end{array}\right\rangle$ is the number of permutations in $\mathfrak{S}_{n}$ with $k-1$ descents, where $1 \leqslant k \leqslant n$ (see [15, A008292]). Let us now recall two classical results.

Proposition 1 ([4, Eq. 4.8]). For $A=\{x, y\}$ and $G=\{x \rightarrow x y, y \rightarrow y\}$, we have

$$
D^{n}(x)=x \sum_{k=1}^{n}\left\{\begin{array}{l}
n \\
k
\end{array}\right\} y^{k} \text { for } n \geqslant 1 .
$$

Proposition 2 ([6, Section 2.1]). For $A=\{x, y\}$ and $G=\{x \rightarrow x y, y \rightarrow x y\}$, we have

$$
D^{n}(x)=\sum_{k=1}^{n}\left\langle\begin{array}{l}
n \\
k
\end{array}\right\rangle x^{k} y^{n-k+1} \quad \text { for } n \geqslant 1 .
$$

One of the most commonly used models of two species predator-prey interaction is the classical Lotka-Volterra system:

$$
\frac{d x}{d t}=x(a-b y), \frac{d y}{d t}=y(-c+d x)
$$

where $y(t)$ and $x(t)$ represent, respectively, the predator population and the prey population as functions of time, and $a, b, c, d$ are positive constants. The differential system (1) is ubiquitous and arises often in mathematical ecology, dynamical system theory and other branches of mathematics (see $[2,3]$ ).

Motivated by (1), we shall consider context-free grammars of the form:

$$
A=\{x, y\}, G=\{x \rightarrow x+p(x, y), y \rightarrow y+q(x, y)\}
$$

where $p(x, y)$ and $q(x, y)$ are polynomials in $x$ and $y$. For convenience, we shall call

$$
G^{\prime}=\{x \rightarrow p(x, y), y \rightarrow q(x, y)\}
$$

the ancestor of $G$.

This paper is a continuation of $[4,6,12]$. Throughout this paper, arrays are indexed by $n, i$ and $j$. Call $\left(a_{n, i, j}\right)$ a combinatorial array if the numbers $a_{n, i, j}$ are nonnegative integers. For any function $H(x, p, q)$, we denote by $H_{y}$ the partial derivative of $H$ with respect to $y$, where $y \in\{x, p, q\}$. In the next section, we present grammatical characterization of some statistics on cyclically ordered partitions. 


\section{Some permutation statistics on cyclically ordered partitions}

Recall that a partition $\pi$ of $[n]$, written $\pi \vdash[n]$, is a collection of disjoint and nonempty subsets $B_{1}, B_{2}, \ldots, B_{k}$ of $[n]$ such that $\bigcup_{i=1}^{k} B_{i}=[n]$, where each $B_{i}(1 \leqslant i \leqslant k)$ is called a block of $\pi$. A cyclically ordered partition of $[n]$ is a partition of $[n]$ whose blocks are endowed with a cyclic order. We always use a canonical representation for cyclically ordered partitions, where the block containing 1 comes first and the integers in each block are in increasing order. For example, (123), (12)(3), (13)(2), (1)(23), (1)(2)(3), (1)(3)(2) are all cyclically ordered partitions of [3]. The opener of a block is its least element. For example, the list of openers of (13)(2) and (1)(3)(2) are respectively given by 12 and 132 . In this section, we shall study some statistics on the list of openers.

\subsection{Descent statistic}

Consider the grammar

$$
G=\{x \rightarrow x+x y, y \rightarrow y+x y\} .
$$

The combinatorial context for the ancestor $G^{\prime}$ of $G$ has been given in Proposition 2 .

From (3), we have

$$
\begin{aligned}
D(x) & =x+x y \\
D^{2}(x) & =x+3 x y+x y^{2}+x^{2} y \\
D^{3}(x) & =x+7 x y+6 x y^{2}+x y^{3}+6 x^{2} y+4 x^{2} y^{2}+x^{3} y
\end{aligned}
$$

For $n \geqslant 0$, we define $D^{n}(x)=\sum_{i \geqslant 1, j \geqslant 0} a_{n, i, j} x^{i} y^{j}$. Since

$$
\begin{aligned}
D^{n+1}(x) & =D\left(\sum_{i, j} a_{n, i, j} x^{i} y^{j}\right) \\
& =\sum_{i, j}(i+j) a_{n, i, j} x^{i} y^{j}+\sum_{i, j} i a_{n, i, j} x^{i} y^{j+1}+\sum_{i, j} j a_{n, i, j} x^{i+1} y^{j}
\end{aligned}
$$

we get

$$
a_{n+1, i, j}=(i+j) a_{n, i, j}+i a_{n, i, j-1}+j a_{n, i-1, j}
$$

for $i, j \geqslant 1$, with the initial conditions $a_{0, i, j}$ to be 1 if $(i, j)=(1,0)$, and to be 0 otherwise. Clearly, $a_{n, 1,0}=1$ and $a_{n, i, 0}=0$ for $i \geqslant 2$.

Example 3. The following table contains the values of $a_{4, i, j}$.

\begin{tabular}{|c|c|c|c|c|c|}
\hline $\boldsymbol{a}_{\mathbf{4}, \boldsymbol{i}, \boldsymbol{j}}$ & $j=0$ & $j=1$ & $j=2$ & $j=3$ & $j=4$ \\
\hline$i=1$ & 1 & 15 & 25 & 10 & 1 \\
$i=2$ & 0 & 25 & 40 & 11 & 0 \\
$i=3$ & 0 & 10 & 11 & 0 & 0 \\
$i=4$ & 0 & 1 & 0 & 0 & 0 \\
\hline
\end{tabular}


Define

$$
A=A(x, p, q)=\sum_{n, i, j \geqslant 0} a_{n, i, j} \frac{x^{n}}{n !} p^{i} q^{j} .
$$

We now present the first main result of this paper.

Theorem 4. The generating function $A$ is given by

$$
A=\frac{p(p-q) e^{x}}{p-q e^{(p-q)\left(e^{x}-1\right)}} .
$$

Moreover, for all $n, i, j \geqslant 1$,

$$
a_{n, i, j}=\left\{\begin{array}{c}
n+1 \\
i+j
\end{array}\right\}\left\langle\begin{array}{c}
i+j-1 \\
i
\end{array}\right\rangle .
$$

Proof. By rewriting (4) in terms of generating function $A$, we have

$$
A_{x}=p(1+q) A_{p}+q(1+p) A_{q} .
$$

It is routine to check that the generating function

$$
\widetilde{A}(x, p, q)=\frac{p(p-q) e^{x}}{p-q e^{(p-q)\left(e^{x}-1\right)}}
$$

satisfies (6). Also, this generating function gives $\widetilde{A}(0, p, q)=p, \widetilde{A}(x, p, 0)=p e^{x}$ and $\widetilde{A}(x, 0, q)=0$ with $q \neq 0$. Hence, $A=\widetilde{A}$. Now let us prove that $a_{n, i, j}=\left\{\begin{array}{c}n+1 \\ i+j\end{array}\right\}\left\langle\begin{array}{c}i+j-1 \\ i\end{array}\right\rangle$. Note that

$$
\begin{aligned}
\frac{d}{d x} \sum_{n, i, k \geqslant 0} a_{n, i, k+1-i} \frac{x^{n+1}}{(n+1) !} v^{i+1} w^{k} & =v \frac{d}{d x} \sum_{k \geqslant 0}\left(\sum_{n \geqslant k+1}\left\{\begin{array}{l}
n+1 \\
k+1
\end{array}\right\} \frac{x^{n+1}}{(n+1) !} \sum_{i=0}^{k}\left\langle\begin{array}{c}
k \\
i
\end{array}\right\rangle v^{i}\right) w^{k} \\
& =v \frac{d}{d x} \sum_{k \geqslant 0}\left(\sum_{i=0}^{k}\left\langle\begin{array}{c}
k \\
i
\end{array}\right\rangle v^{i}\right) \frac{\left(e^{x}-1\right)^{k+1}}{(k+1) !} w^{k} .
\end{aligned}
$$

By using the fact that

$$
\sum_{k \geqslant 0}\left(\sum_{i=0}^{k}\left\langle\begin{array}{c}
k \\
i
\end{array}\right) p^{i}\right) u^{k}=\int_{0}^{u} \frac{p-1}{p-e^{u^{\prime}(p-1)}} d u^{\prime}=\frac{1}{p}\left(u(p-1)-\ln \left(e^{u(p-1)}-p\right)+\ln (1-p)\right),
$$

we obtain that

$$
v \frac{d}{d x} \sum_{n, i, k \geqslant 0} a_{n, i, k+1-i} \frac{x^{n+1}}{(n+1) !} v^{i} w^{k}=\frac{w v(v-1) e^{x}}{v-e^{\left(e^{x}-1\right) w(v-1)}}
$$

which implies

$$
A(x, v w, w)=\frac{w v(v-1) e^{x}}{v-e^{\left(e^{x}-1\right) w(v-1)}}
$$

as required. 
Let $a_{n}=\sum_{i \geqslant 1, j \geqslant 0} a_{n, i, j}$. Clearly, $a_{n}=\sum_{k=0}^{n} k !\left\{\begin{array}{c}n+1 \\ k+1\end{array}\right\}$.

Proposition 5. $\left\{\begin{array}{c}n \\ k\end{array}\right\}\left\langle\begin{array}{c}k-1 \\ i\end{array}\right\rangle$ is the number of cyclically ordered partitions of [n] with $k$ blocks whose list of openers contains $i-1$ descents.

Proof. To form such a cyclically ordered partition, start with a partition of $[n]$ into $k$ blocks in canonical form, each block increasing and blocks arranged in order of increasing first entries (there are $\left\{\begin{array}{l}n \\ k\end{array}\right\}$ choices). The first opener is thus 1. Then leave the first block in place and rearrange the $k-1$ remaining blocks so that their openers, viewed as a list, contain $i-1$ descents (there are $\left\langle\begin{array}{c}k-1 \\ i\end{array}\right\rangle$ choices).

We can now conclude the following corollary from the discussion above.

Corollary 6. For all $n, i, j \geqslant 1, a_{n, i, j}$ is the number of cyclically ordered partitions of $[n+1]$ with $i+j$ blocks whose list of openers contains $i-1$ descents.

\subsection{Peak statistics}

The idea of a peak (resp. valley) in a list of integers $\left(w_{i}\right)_{i=1}^{n}$ is an entry that is greater (resp. smaller) than its neighbors. The number of peaks in a permutation is an important combinatorial statistic. See, e.g., $[1,5,7,10]$ and the references therein. However, the question of whether the first and/or last entry may qualify as a peak (or valley) gives rise to several different definitions. In this paper, we consider only left peaks and right valleys. A left peak index is an index $i \in[n-1]$ such that $w_{i-1}<w_{i}>w_{i+1}$, where we take $w_{0}=0$, and the entry $w_{i}$ is a left peak. Similarly, a right valley is an entry $w_{i}$ with $i \in[2, n]$ such that $w_{i-1}>w_{i}<w_{i+1}$, where we take $w_{n+1}=\infty$. Thus the last entry may be a right valley but not a left peak. For example, the list 64713258 has 3 left peaks and 3 right valleys. Clearly, left peaks and right valleys in a list are equinumerous: they alternate with a peak first and a valley last. Peaks and valleys were considered in [7]. The left peak statistic first appeared in [1, Definition 3.1].

Let $P(n, k)$ be the number of permutations in $\mathfrak{S}_{n}$ with $k$ left peaks. Let $P_{n}(x)=$ $\sum_{k \geqslant 0} P(n, k) x^{k}$. It is well known [15, A008971] that

$$
\begin{aligned}
P(x, z) & =1+\sum_{n \geqslant 1} P_{n}(x) \frac{z^{n}}{n !} \\
& =\frac{\sqrt{1-x}}{\sqrt{1-x} \cosh (z \sqrt{1-x})-\sinh (z \sqrt{1-x})}
\end{aligned}
$$

Let $D$ be the differential operator $\frac{d}{d \theta}$. Set $x=\sec \theta$ and $y=\tan \theta$. Then

$$
D(x)=x y, D(y)=x^{2} .
$$

Furthermore, if $G^{\prime}=\left\{x \rightarrow x y, y \rightarrow x^{2}\right\}$, then

$$
D_{G^{\prime}}^{n}(x)=\sum_{k=0}^{\left\lfloor\frac{n}{2}\right\rfloor} P(n, k) x^{2 k+1} y^{n-2 k} \quad \text { for } n \geqslant 1,
$$


which was given in [10, Section 2]. There is a large literature devoted to the repeated differentiation of the secant and tangent functions (see $[8,9,10,11]$ for instance).

Consider the grammar

$$
G=\left\{x \rightarrow x+x y, y \rightarrow y+x^{2}\right\} .
$$

From (7), we have

$$
\begin{aligned}
D(x) & =x+x y \\
D^{2}(x) & =x+3 x y+x y^{2}+x^{3}, \\
D^{3}(x) & =x+7 x y+6 x y^{2}+x y^{3}+6 x^{3}+5 x^{3} y .
\end{aligned}
$$

Define

$$
D^{n}(x)=\sum_{i \geqslant 1, j \geqslant 0} b_{n, i, j} x^{i} y^{j}
$$

Since

$$
\begin{aligned}
D^{n+1}(x) & =D\left(\sum_{i \geqslant 1, j \geqslant 0} b_{n, i, j} x^{i} y^{j}\right) \\
& =\sum_{i, j}(i+j) b_{n, i, j} x^{i} y^{j}+\sum_{i, j} i b_{n, i, j} x^{i} y^{j+1}+\sum_{i, j} j b_{n, i, j} x^{i+2} y^{j-1},
\end{aligned}
$$

we get

$$
b_{n+1, i, j}=(i+j) b_{n, i, j}+i b_{n, i, j-1}+(j+1) b_{n, i-2, j+1}
$$

for $i \geqslant 1$ and $j \geqslant 0$, with the initial conditions $b_{0, i, j}$ to be 1 if $(i, j)=(1,0)$, and to be 0 otherwise. Clearly, $b_{n, 1,0}=1$ for $n \geqslant 1$.

Example 7. The following table contains the values of $b_{4, i, j}$.

\begin{tabular}{|c|c|c|c|c|c|}
\hline $\boldsymbol{b}_{\mathbf{4}, \boldsymbol{i}, \boldsymbol{j}}$ & $j=0$ & $j=1$ & $j=2$ & $j=3$ & $j=4$ \\
\hline$i=1$ & 1 & 15 & 25 & 10 & 1 \\
$i=3$ & 25 & 50 & 18 & 0 & 0 \\
$i=5$ & 5 & 0 & 0 & 0 & 0 \\
\hline
\end{tabular}

Define

$$
B=B(x, p, q)=\sum_{n, i, j \geqslant 0} b_{n, i, j} p^{i} q^{j} \frac{x^{n}}{n !} .
$$

We now present the second main result of this paper.

Theorem 8. The generating function $B$ is given by

$$
B(x, p, q)=\frac{p \sqrt{q^{2}-p^{2}} e^{x}}{\sqrt{q^{2}-p^{2}} \cosh \left(\sqrt{q^{2}-p^{2}}\left(e^{x}-1\right)\right)-q \sinh \left(\sqrt{q^{2}-p^{2}}\left(e^{x}-1\right)\right)} .
$$

Moreover, for all $n, i, j \geqslant 1$,

$$
b_{n, 2 i-1, j}=\left\{\begin{array}{c}
n+1 \\
2 i-1+j
\end{array}\right\} P(2 i-2+j, i-1) .
$$


Proof. The recurrence (8) can be written as

$$
B_{x}=p(1+q) B_{p}+\left(p^{2}+q\right) B_{q} .
$$

It is routine to check that the generating function

$$
\widetilde{B}=\widetilde{B}(x, p, q)=\frac{p \sqrt{q^{2}-p^{2}} e^{x}}{\sqrt{q^{2}-p^{2}} \cosh \left(\sqrt{q^{2}-p^{2}}\left(e^{x}-1\right)\right)-q \sinh \left(\sqrt{q^{2}-p^{2}}\left(e^{x}-1\right)\right)}
$$

satisfies (10)). Also, this generating function gives $\widetilde{B}(0, p, q)=p$ and $\widetilde{B}(x, 0, q)=0$. Hence, $B=\widetilde{B}$.

It follows from $(8)$ that $b_{n, 2 i, j}=0$ for all $(i, j) \neq(0,0)$. Now let us prove that

$$
b_{n, 2 i-1, j}=\left\{\begin{array}{c}
n+1 \\
2 i-1+j
\end{array}\right\} P(2 i-2+j, i-1) .
$$

Note that

$$
\begin{aligned}
\sum_{n, i, j \geqslant 0} b_{n, i, j+1-2 i} p^{i} q^{j} \frac{x^{n}}{n !} & =\sum_{n \geqslant 0, i, j \geqslant 1} b_{n, 2 i-1, j+1-2 i} p^{i} q^{j} \frac{x^{n}}{n !}=p \sum_{n \geqslant 0, j \geqslant 1}\left\{\begin{array}{c}
n+1 \\
j
\end{array}\right\} P_{j-1}(p) q^{j} \frac{x^{n}}{n !} \\
& =p e^{x} \sum_{j \geqslant 1} \frac{\left(e^{x}-1\right)^{j-1}}{(j-1) !} P_{j-1}(p) q^{j}=p q e^{x} P\left(p, q\left(e^{x}-1\right)\right),
\end{aligned}
$$

Hence,

$$
\sum_{n, i, j \geqslant 0} b_{n, i, j} p^{i} q^{j} \frac{x^{n}}{n !}=p e^{x} P\left(p^{2} / q^{2}, q\left(e^{x}-1\right)\right)=B(x, p, q)
$$

as required.

Let $b_{n}=\sum_{i \geqslant 1, j \geqslant 0} b_{n, i, j}$. It follows from (9) that $b_{n}=a_{n}$. In the following discussion, we shall present a combinatorial interpretation for $b_{n, i, j}$.

Lemma 9. Suppose that $\left(w_{i}\right)_{i=1}^{k}$ is a list of distinct integers containing $\ell$ right valleys and that $w_{1}=1$. Then, among the $k$ ways to insert a new entry $m>\max \left(w_{i}\right)$ into the list in a noninitial position, $2 \ell+1$ of them will not change the number of right valleys and $k-(2 \ell+1)$ will increase it by 1 .

Proof. As observed above, peaks and valleys alternate, a peak occurring first, and a valley occurring last. Thus there are $\ell$ peaks. If $m$ is inserted immediately before or after a peak or at the very end, the number of valleys is unchanged, otherwise it is increased by 1 .

Proposition 10. The number $u_{n, k, \ell}$ of cyclically ordered partitions on $[n]$ with $k$ blocks and $\ell$ right valleys in the list of openers satisfies the recurrence

$$
u_{n, k, \ell}=k u_{n-1, k, \ell}+(2 \ell+1) u_{n-1, k-1, \ell}+(k-2 \ell) u_{n-1, k-1, \ell-1}
$$

for $n \geqslant 2, \ell \geqslant 0,2 \ell+1 \leqslant k \leqslant n$. 
Proof. Each cyclically ordered partition of size $n$ is obtained by inserting $n$ into one of size $n-1$, either as the last entry in an existing block or as a new singleton block. Let $\mathcal{U}_{n, k, \ell}$ denote the set of cyclically ordered partitions counted by $u_{n, k, \ell}$. To obtain an element of $\mathcal{U}_{n, k, \ell}$ we can insert $n$ into any existing block of an element of $\mathcal{U}_{n-1, k, \ell}$ (this gives $k u_{n-1, k, \ell}$ choices ), or insert $n$ as a singleton block into an element of $\mathcal{U}_{n-1, k-1, \ell}$ so that the number of right valleys is unchanged (this gives $(2 \ell+1) u_{n-1, k-1, \ell}$ choices ), or insert $n$ as a singleton block into an element of $\mathcal{U}_{n-1, k-1, \ell-1}$ so that the number of right valleys is increased by 1 (this gives $(k-2 \ell) u_{n-1, k-1, \ell-1}$ choices ). The last two counts of choices follow from Lemma 9.

Corollary 11. For all $n, i, j \geqslant 1, b_{n, i, j}$ is the number of cyclically ordered partitions of $[n+1]$ with $i+j$ blocks and $\frac{i-1}{2}$ right valleys (equivalently, $\frac{i-1}{2}$ left peaks) in the list of openers.

Proof. Comparing recurrence relations (8) and (11), we see that $b_{n, i, j}=u_{n+1, i+j,(i-1) / 2}$.

Remark 12. A cyclically ordered partition of size $n$ with $k$ blocks and $\ell$ right valleys in the list of openers is obtained by selecting a partition of $[n]$ with $k$ blocks in $\left\{\begin{array}{l}n \\ k\end{array}\right\}$ ways, and then arranging the blocks suitably, in $P(k, \ell)$ ways. Hence $u_{n, k, \ell}=\left\{\begin{array}{l}n \\ k\end{array}\right\} P(k, \ell)$ and we get a combinatorial proof that $b_{n, 2 i-1, j}=\left\{\begin{array}{c}n+1 \\ 2 i-1+j\end{array}\right\} P(2 i-2+j, i-1)$.

\subsection{The longest alternating subsequences}

Let $\pi=\pi(1) \pi(2) \cdots \pi(n) \in \mathfrak{S}_{n}$. An alternating subsequence of $\pi$ is a subsequence $\pi\left(i_{1}\right) \cdots \pi\left(i_{k}\right)$ satisfying

$$
\pi\left(i_{1}\right)>\pi\left(i_{2}\right)<\pi\left(i_{3}\right)>\cdots \pi\left(i_{k}\right)
$$

Let as $(\pi)$ be the length (number of terms) of the longest alternating subsequence of $\pi$. Denote by $a_{k}(n)$ the number of permutations $\pi$ in $\mathfrak{S}_{n}$ such that as $(\pi)=k$. The study of the distribution of the length of the longest alternating subsequences of permutations was recently initiated by Stanley [16].

Let $L_{n}(x)=\sum_{k=0}^{n} a_{k}(n) x^{k}$, and let

$$
L(x, z)=\sum_{n \geqslant 0} L_{n}(x) \frac{z^{n}}{n !} .
$$

Stanley [16, Theorem 2.3] obtained the following closed-form formula:

$$
L(x, z)=(1-x) \frac{1+\rho+2 x e^{\rho z}+(1-\rho) e^{2 \rho z}}{1+\rho-x^{2}+\left(1-\rho-x^{2}\right) e^{2 \rho z}}
$$

where $\rho=\sqrt{1-x^{2}}$.

Let $\pi=\pi(1) \pi(2) \cdots \pi(n) \in \mathfrak{S}_{n}$. We say that $\pi$ changes direction at position $i$ if either $\pi(i-1)<\pi(i)>\pi(i+1)$, or $\pi(i-1)>\pi(i)<\pi(i+1)$, where $i \in\{2,3, \ldots, n-1\}$. We say that $\pi$ has $k$ alternating runs if there are $k-1$ indices $i$ such that $\pi$ changes direction 
at these positions. The up-down runs of a permutation $\pi$ are the alternating runs of $\pi$ endowed with a 0 in the front. For example, the permutation $\pi=514632$ has 3 alternating runs and 4 up-down runs. One can easily verify that $a_{k}(n)$ also counts permutations in $\mathfrak{S}_{n}$ with $k$ up-down runs. It follows from [13, Corollary 8] that

$$
L(x, z)=-\sqrt{\frac{x-1}{x+1}}\left(\frac{\sqrt{x^{2}-1}+x \sin \left(z \sqrt{x^{2}-1}\right)}{1-x \cos \left(z \sqrt{x^{2}-1}\right)}\right) .
$$

Set $P_{0}(x)=L_{0}(x)=1$. There is a closely connection between the polynomials $P_{n}(x)$ and $L_{n}(x)$ (see [13, Corollary 7]):

$$
L_{n+1}(x)=x \sum_{k=0}^{n}\left(\begin{array}{l}
n \\
k
\end{array}\right) L_{k}(x) P_{n-k}\left(x^{2}\right) .
$$

We now present a grammatical characterization of the numbers $a_{k}(n)$.

Proposition 13 ([13, Theorem 6]). For $A=\{w, x, y\}$ and $G^{\prime}=\{w \rightarrow w x, x \rightarrow x y, y \rightarrow$ $\left.x^{2}\right\}$, we have

$$
D_{G^{\prime}}^{n}(w)=w \sum_{k=0}^{n} a_{k}(n) x^{k} y^{n-k} .
$$

Consider the grammar

$$
G=\left\{w \rightarrow w+w x, x \rightarrow x+x y, y \rightarrow y+x^{2}\right\},
$$

which is the descendant of $G^{\prime}$ introduced in Proposition 13. From (13), we have

$$
\begin{aligned}
D(w) & =w(1+x), \\
D^{2}(w) & =w\left(1+3 x+x y+x^{2}\right) ; \\
D^{3}(w) & =w\left(1+7 x+6 x y+x y^{2}+6 x^{2}+3 x^{2} y+2 x^{3}\right) .
\end{aligned}
$$

Define

$$
D^{n}(w)=w \sum_{i, j \geqslant 0} t_{n, i, j} x^{i} y^{j}
$$

Since

$$
\begin{aligned}
& D^{n+1}(w) \\
& =D\left(w \sum_{i, j \geqslant 0} t_{n, i, j} x^{i} y^{j}\right) \\
& =\sum_{i, j}(1+i+j) t_{n, i, j} x^{i} y^{j}+\sum_{i, j} t_{n, i, j} x^{i+1} y^{j}+\sum_{i, j} i t_{n, i, j} x^{i} y^{j+1}+\sum_{i, j} j t_{n, i, j} x^{i+2} y^{j-1},
\end{aligned}
$$

we get

$$
t_{n+1, i, j}=(1+i+j) t_{n, i, j}+t_{n, i-1, j}+i t_{n, i, j-1}+(j+1) t_{n, i-2, j+1}
$$

for $i, j \geqslant 0$, with the initial conditions $t_{0, i, j}$ to be 1 if $(i, j)=(0,0)$ or $(i, j)=(1,0)$, and to be 0 otherwise. Clearly, $t_{n, 0,0}=1$ for $n \geqslant 0$. 
Example 14. The following table contains the values of $t_{4, i, j}$.

\begin{tabular}{|c|c|c|c|c|}
\hline $\boldsymbol{t}_{\mathbf{4}, \boldsymbol{i}, \boldsymbol{j}}$ & $j=0$ & $j=1$ & $j=2$ & $j=3$ \\
\hline$i=0$ & 1 & 0 & 0 & 0 \\
$i=1$ & 15 & 25 & 10 & 1 \\
$i=2$ & 25 & 30 & 7 & 0 \\
$i=3$ & 20 & 11 & 0 & 0 \\
$i=4$ & 5 & 0 & 0 & 0 \\
\hline
\end{tabular}

Define

$$
T=T(x, p, q)=\sum_{n, i, j \geqslant 0} t_{n, i, j} p^{i} q^{j} \frac{x^{n}}{n !} .
$$

We now present the following.

Theorem 15. The generating function $T$ is given by

$$
T(x, p, q)=e^{x} \sqrt{\frac{p-q}{p+q}} \frac{\sqrt{p^{2}-q^{2}}+p \sin \left(\left(e^{x}-1\right) \sqrt{p^{2}-q^{2}}\right)}{p \cos \left(\left(e^{x}-1\right) \sqrt{p^{2}-q^{2}}\right)-q} .
$$

Moreover, for all $n \geqslant 1, i \geqslant 1$ and $j \geqslant 0$,

$$
t_{n, i, j}=\left\{\begin{array}{c}
n+1 \\
i+j+1
\end{array}\right\} a_{i}(i+j) .
$$

Proof. The recurrence (14) can be written as

$$
T_{x}=T+p(1+q) T_{p}+\left(p^{2}+q\right) T_{q} .
$$

It is routine to check that the generating function

$$
\widetilde{T}=\widetilde{T}(x, p, q)=e^{x} \sqrt{\frac{p-q}{p+q}} \frac{\sqrt{p^{2}-q^{2}}+p \sin \left(\left(e^{x}-1\right) \sqrt{p^{2}-q^{2}}\right)}{p \cos \left(\left(e^{x}-1\right) \sqrt{p^{2}-q^{2}}\right)-q}
$$

satisfies (16)). Also, this generating function gives $\widetilde{T}(0, p, q)=1$ and $\widetilde{T}(x, 0, q)=e^{x}$. Hence, $T=\widetilde{T}$.

Now let us prove that $t_{n, 2 i-1, j}=\left\{\begin{array}{c}n+1 \\ i+j+1\end{array}\right\} a_{i}(i+j)$. Note that

$$
\begin{aligned}
\sum_{n, i, j \geqslant 0} t_{n, i, j-i} p^{i} q^{j} \frac{x^{n}}{n !} & =\sum_{n, i, j \geqslant 0} t_{n, i, j-i} p^{i} q^{j} \frac{x^{n}}{n !}=\sum_{n, j \geqslant 0}\left\{\begin{array}{c}
n+1 \\
j+1
\end{array}\right\} L_{j}(p) q^{j} \frac{x^{n}}{n !} \\
& =e^{x} \sum_{j \geqslant 0} \frac{\left(e^{x}-1\right)^{j}}{(j) !} L_{j}(p) q^{j}=e^{x} L\left(p, q\left(e^{x}-1\right)\right),
\end{aligned}
$$

Hence,

$$
\sum_{n, i, j \geqslant 0} t_{n, i, j} p^{i} q^{j} \frac{x^{n}}{n !}=e^{x} L\left(p / q, q\left(e^{x}-1\right)\right)=T(x, p, q),
$$

as required. 
Let $t_{n}=\sum_{i \geqslant 1, j \geqslant 0} t_{n, i, j}$. It follows from (15) that $t_{n}=\sum_{k=0}^{n} k !\left\{\begin{array}{l}n+1 \\ k+1\end{array}\right\}$. Along the same lines as the proof of Corollary 6 , we get the following.

Corollary 16. For all $n \geqslant 1, i \geqslant 1$ and $j \geqslant 0, t_{n, i, j}$ is the number of cyclically ordered partitions of $[n+1]$ having $i+j+1$ blocks such that the list of openers has the longest alternating subsequence of length $i$.

\section{Concluding remarks}

In this paper, we explore some context-free grammars suggested by (1). In fact, there are many other extension of (1). For example, many authors investigated the following generalized Lotka-Volterra system (see [14]):

$$
\frac{d x}{d t}=x(C y+z), \frac{d y}{d t}=y(A z+x), \frac{d z}{d t}=z(B x+y) .
$$

Consider the grammar

$$
G=\{x \rightarrow x(y+z), y \rightarrow y(z+x), z \rightarrow z(x+y)\}
$$

Define

$$
D^{n}(x)=\sum_{i \geqslant 1, j \geqslant 0} g_{n, i, j} x^{i} y^{j} z^{n+1-i-j} .
$$

By induction, one can easily verify the following: for all $n \geqslant 1, i \geqslant 1$ and $j \geqslant 0$, we have

$$
g_{n, i, 0}=\left\langle\begin{array}{c}
n \\
i
\end{array}\right\rangle, g_{n, i, n+1-i}=\left\langle\begin{array}{c}
n \\
i
\end{array}\right\rangle, g_{n, 1, j}=\left\langle\begin{array}{c}
n+1 \\
j+1
\end{array}\right\rangle .
$$

\section{Acknowledgements}

The authors thank the referee for many detailed suggestions leading to a substantial improvement of this paper.

\section{References}

[1] M. Aguiar, N. Bergeron, K. Nyman. The peak algebra and the descent algebras of types B and D. Trans. Amer. Math. Soc., 356(7): 2781-2824, 2004.

[2] L.J.S. Allen. An Introduction to Mathematical Biology. Prentice Hall, New Jersey, 2007.

[3] E. Chauvet, J.E. Paullet, J.P. Previte, Z. Walls. A Lotka-Volterra Three-species Food Chain. Math. Mag., 75: 243-255, 2002.

[4] W.Y.C. Chen. Context-free grammars, differential operators and formal power series. Theoret. Comput. Sci., 117: 113-129, 1993. 
[5] K. Dilks, T.K. Petersen, J.R. Stembridge. Affine descents and the Steinberg torus. Adv. in Appl. Math., 42: 423-444, 2009.

[6] D. Dumont. Grammaires de William Chen et dérivations dans les arbres et arborescences. Sém. Lothar. Combin., 37, Art. B37a: 1-21, 1996.

[7] J. Françon and G. Viennot. Permutations selon leurs pics, creux, doubles montées et double descentes, nombres d'Euler et nombres de Genocchi. Discrete Math., 28: 21-35, 1979.

[8] G. R. Franssens. Functions with derivatives given by polynomials in the function itself or a related function. Anal. Math., 33: 17-36, 2007.

[9] M. E. Hoffman. Derivative polynomials, Euler polynomials, and associated integer sequences. Electron. J. Combin., 6: \#R21, 1999.

[10] S.-M. Ma. Derivative polynomials and enumeration of permutations by number of interior and left peaks. Discrete Math., 312: 405-412, 2012.

[11] S.-M. Ma. A family of two-variable derivative polynomials for tangent and secant. Electron. J. Combin., 20(1): \#P11, 2013.

[12] S.-M. Ma. Some combinatorial arrays generated by context-free grammars. European J. Combin., 34: 1081-1091, 2013.

[13] S.-M. Ma. Enumeration of permutations by number of alternating runs. Discrete Math., 313: 1816-1822, 2013.

[14] J.M. Ollagnier. Liouvillian integration of the Lotka-Volterra system. Qual. Theory Dyn. Syst., 2: 307-358, 2001.

[15] N.J.A. Sloane. The On-Line Encyclopedia of Integer Sequences, 2010. http://oeis . org

[16] R.P. Stanley. Longest alternating subsequences of permutations. Michigan Math. J., 57: 675-687, 2008. 